\title{
Modified energetics and growth kinetics on H-terminated GaAs (110)
}

\author{
B. Galiana, ${ }^{1,2}$ M. Benedicto, ${ }^{1}$ L. Díez-Merino, ${ }^{1}$ S. Lorbek, ${ }^{3}$ G. Hlawacek, ${ }^{3}$ C. Teichert, ${ }^{3}$ \\ and P. Tejedor ${ }^{1, a)}$ \\ ${ }^{1}$ Instituto de Ciencia de Materiales de Madrid, CSIC, Sor Juana Inés de la Cruz 3, 28049 Madrid, Spain \\ ${ }^{2}$ Departamento de Física, Universidad Carlos III de Madrid, Avenida de la Universidad 30, \\ 28911 Madrid, Spain \\ ${ }^{3}$ Institut für Physik, Montanuniversität Leoben, Franz Josef St., 18A-8700 Leoben, Austria
}

(Received 27 May 2013; accepted 4 October 2013; published online 29 October 2013)

\begin{abstract}
Atomic hydrogen modification of the surface energy of GaAs (110) epilayers, grown at high temperatures from molecular beams of $\mathrm{Ga}$ and $\mathrm{As}_{4}$, has been investigated by friction force microscopy (FFM). The reduction of the friction force observed with longer exposures to the $\mathrm{H}$ beam has been correlated with the lowering of the surface energy originated by the progressive de-relaxation of the GaAs (110) surface occurring upon $\mathrm{H}$ chemisorption. Our results indicate that the H-terminated GaAs (110) epilayers are more stable than the As-stabilized ones, with the minimum surface energy value of $31 \mathrm{meV} / \AA^{2}$ measured for the fully hydrogenated surface. A significant reduction of the $\mathrm{Ga}$ diffusion length on the $\mathrm{H}$-terminated surface irrespective of $\mathrm{H}$ coverage has been calculated from the FFM data, consistent with the layer-by-layer growth mode and the greater As incorporation coefficient determined from real-time reflection high-energy electron diffraction studies. Arsenic incorporation through direct dissociative chemisorption of single $\mathrm{As}_{4}$ molecules mediated by $\mathrm{H}$ on the GaAs (110) surface has been proposed as the most likely explanation for the changes in surface kinetics observed. (C) 2013 AIP Publishing LLC. [http://dx.doi.org/10.1063/1.4826452]
\end{abstract}

\section{INTRODUCTION}

Semiconductor epitaxy using surface active species (surfactants), such as $\mathrm{H}, \mathrm{As}, \mathrm{Sb}, \mathrm{Te}$, or $\mathrm{Sn}$, has attracted much interest during the last decades, mainly due to the possibility to suppress three-dimensional (3D) island growth for device applications. $^{1-4}$ In the particular case of III-V semiconductor molecular beam epitaxy (MBE) on (110)-oriented GaAs substrates, which finds application in the field of nonmagnetic spintronics, ${ }^{5}$ it has been reported that the use of atomic hydrogen as a surfactant alters the kinetics of adatom incorporation to step edges, leading to two-dimensional (2D) layerby-layer growth of GaAs and 3D growth of InGaAs/GaAs quantum dots at low temperatures. ${ }^{6-8}$ But the effect of this surfactant on the epilayer surface energy was not addressed in these studies. A deeper understanding on how a surfactant species influences the semiconductor growth mode can be attained through a combined investigation of both thermodynamics, i.e., surface and interface energies, and kinetics of adatom migration and incorporation to island/step edges. Reflection high energy electron diffraction (RHEED) provides a unique means to study in real time the dynamics of III-V growth during the MBE process, mainly through the quantitative analysis of the so-called intensity oscillations of the specular beam in the diffraction pattern, whose period corresponds to the growth of a single monolayer (ML) on a (001) substrate. The RHEED oscillatory behaviour has been explained in terms of a temporal variation in the surface step edge density that results from 2D layer-by-layer growth. In

\footnotetext{
a) Author to whom correspondence should be addressed. Electronic mail: ptejedor@icmm.csic.es
}

contrast with GaAs growth on the (001) surface, which is kinetically controlled by the migration of the Ga adatoms to the active sites where the reaction with As takes place, growth on (110)-oriented substrates is limited by the chemical reaction between $\mathrm{Ga}$ and $\mathrm{As}$, given that the lifetime of the group $\mathrm{V}$ species on this surface tends to be very short. In consequence, the RHEED oscillation technique can be applied on this surface to measure directly the incorporation kinetics of the group $\mathrm{V}$ element. ${ }^{9,10}$

Friction force microscopy (FFM), also known as lateral force microscopy or chemical force microscopy, has proved to be a useful technique to estimate variations in surface energy due to the presence of different termination groups on epitaxially grown thin films. In this technique an atomic force microscope equipped with a beam deflection system and a quadrant photodiode detector is used to measure both the vertical and the lateral forces, the latter corresponding to the frictional force exerted by a nanoscale tip on a "single asperity" moving relative to the surface under study. Based on the fact that the lateral force acting on the tip is influenced by its friction coefficient on the sample surface, which in turn is very sensitive to the terminating group at the thin film surface, the reduction in friction observed experimentally is directly related to a lowering of the surface energy $\left(E_{\text {surf }}\right)$, as established by Hlawacek ${ }^{11}$ and Skulason. ${ }^{12}$

In this work we present a combined FFM and RHEED study on the variations in surface energy and adatom kinetics that occur during surfactant-aided molecular beam homoepitaxy of GaAs on vicinal (110)-oriented substrates. Specifically, we show that exposure to an atomic hydrogen beam prior or during growth decreases the GaAs(110) surface energy as a result of the surface counter-relaxation caused by 
hydrogen chemisorption. In addition, real-time dynamic studies of the growth kinetics demonstrate that atomic $\mathrm{H}$ facilitates the dissociative chemisorption of $\mathrm{As}_{4}$, used as the arsenic source, leading to a reduction of the effective Ga diffusion length on the H-terminated (110) surface and a layer-by-layer growth mode.

\section{EXPERIMENTAL DETAILS}

The GaAs samples studied in this work were grown in a modified solid-source VARIAN $360 \mathrm{MBE}$ system. Ga and $\mathrm{As}_{4}$ fluxes were calibrated using RHEED intensity oscillations on a GaAs (001) substrate. Atomic hydrogen was generated in the growth chamber by dissociation of $\mathrm{H}_{2}$ gas at a W filament heated to a temperature of $1800^{\circ} \mathrm{C}$. Epiready GaAs (110) substrates misoriented $2^{\circ}$ towards (111)A were indium bonded to Mo disks and outgassed at $350{ }^{\circ} \mathrm{C}$ before the surface oxide was removed by thermal desorption at a substrate temperature of $620^{\circ} \mathrm{C}$ under an $\mathrm{As}_{4}$ flux of 2.5 $\times 10^{15}$ molecules $\mathrm{cm}^{-2} \mathrm{~s}^{-1}$. Alternatively, the native oxide was removed by reaction with atomic hydrogen at $450^{\circ} \mathrm{C}$ for 20 min, using a concomitant $\mathrm{As}_{4}$ beam. After oxide removal, 60 nm-thick GaAs layers $(1 \mathrm{ML}=2 \AA$ for GaAs (110)) were deposited at a substrate temperature of $620^{\circ} \mathrm{C}$ with an As/Ga flux ratio of 10 . The nominal growth rate was $0.2 \mathrm{ML}$ $\mathrm{s}^{-1}$. Three different samples were prepared in order to study the effect of atomic hydrogen at different steps of the process, using the following procedures: (i) thermal desorption of the surface oxide and conventional MBE growth (sample I), (ii) $\mathrm{H}$-assisted oxide desorption and conventional $\mathrm{MBE}$ growth (sample II), and (iii) $\mathrm{H}$-assisted oxide desorption followed by $\mathrm{H}$-assisted MBE growth (sample III). The background pressure during $\mathrm{H}$-assisted oxide cleaning and $\mathrm{H}$ assisted growth was $\sim 2 \times 10^{-6}$ Torr. Growth dynamics on the GaAs (110) vicinal surface were investigated in situ by recording the oscillatory behaviour of the RHEED specular beam intensity along the main step edge direction, i.e., [11 0 ] azimuth at a very low incident polar angle $\left(<1^{\circ}\right)$, under identical conditions to those used to deposit samples I, II, and III.

The surface morphology of the GaAs (110) layers was examined by AFM using a Digital Instruments-Multimode IIIa microscope working in tapping mode. Conventional $\mathrm{Si}$ cantilevers with a typical resonance frequency of $300 \mathrm{kHz}$ were used as tips. The average value of the terrace width was calculated from a set of over 200 cross-section profiles taken along the [001] tilt direction for each sample. The surface free energy of the GaAs epilayers was measured by FFM using the same microscope in contact mode. A specially designed rectangular beam type $\mathrm{Si}$ cantilever was used for this purpose. The cantilever had a nominal length of $225 \mu \mathrm{m}$ and a typical force constant of $0.2 \mathrm{~N} / \mathrm{m}$. Friction measurements were performed by imaging with constant force and recording the lateral deflection of the cantilever in the direction of the scanning motion (i.e., perpendicular to its long axis), thus providing the friction image. ${ }^{13,14}$ The torsion of the cantilever induced by friction leads to a movement of the laser on the position sensitive photodiode in the lateral direction, causing a change in the voltage signal, $\left(\Delta V_{p h}\right)$. Applying Hooke's law, the raw voltage data can be converted to friction force:

$$
F_{\text {lat }}=k_{\text {tor }} \frac{\Delta V_{p h}}{S_{\text {lat }}}
$$

where $k_{t o r}$ is the torsion lateral spring constant and $S_{l a t}$ is the lateral sensitivity of the photodiode, respectively. Since $\Delta V_{p h}$ is proportional to $F_{\text {lat }}$, the raw voltage data allow establishing a hierarchy of friction forces, regardless of the $k_{t o r}$ and $\mathrm{S}_{\text {lat }}$ values. Therefore, relative changes in friction between samples can be determined quite accurately by using the same cantilever and tip during all measurements, as demonstrated by several authors. ${ }^{4,15}$ In this work the $\Delta V_{p h}$ data extracted for each sample were the average values of six separate FFM measurements obtained from different surface regions. These measurements were typically performed in $1 \times 1 \mu \mathrm{m}^{2}$ scan areas. Images were obtained in the usual topographic mode by maintaining a constant applied normal force, while simultaneously recording the lateral force signal. Each of the individual data points was obtained from a friction loop, i.e., the $\Delta V_{p h}$ value was calculated from the average difference in bidirectional lateral force signals: (forward scan - reverse scan)/2. After the FFM image was obtained, the average value of $\Delta V_{p h}$ was calculated from each scan to minimize the effect of the steps in the raw voltage data.

\section{RESULTS AND DISCUSSION}

Figure 1 depicts $1 \mu \mathrm{m} \times 1 \mu \mathrm{m}$ AFM topography images and their corresponding friction force microscopy images for the GaAs(110) vicinal surface after deposition of $60 \mathrm{~nm} \mathrm{GaAs}$ by (a) conventional MBE growth (sample I), (b) $\mathrm{H}$-assisted oxide desorption followed by conventional MBE growth (sample II), and (c) H-assisted oxide desorption and H-MBE growth (sample III).

The AFM images show a characteristic faceting of the surface with straight bunched steps along the [110] direction for all three samples. The progressive narrowing of the terrace width and tendency towards step debunching with increasing atomic $\mathrm{H}$ dose previously reported ${ }^{16,17}$ can be clearly observed in these images. In addition, the rms surface roughness decreases after exposure to the atomic hydrogen beam, this effect being more pronounced when the exposure is continued during epitaxial growth. The corresponding $\Delta V_{p h}$ colormaps obtained from the FFM measurements are presented underneath each image. The maps evidence a marked influence of the step edges on the FFM signal. In order to minimize this effect and enhance the contribution from the terrace surface, we have worked with large scan sizes and calculated the average $\Delta V_{p h}$ values according to the procedure described above. The data have then been normalized with respect to the conventional MBE grown GaAs (110) sample to facilitate their analysis. As shown in Table I, when GaAs (110) homoepitaxy is carried out on the H-cleaned vicinal surface (sample II) the value of $\Delta V_{p h}$ undergoes a reduction of $18 \%$ with respect to the sample grown by conventional MBE (sample I). Moreover, the measured reduction amounts to $30 \%$ when the surface is exposed to the atomic $\mathrm{H}$ beam during growth too (sample III). Therefore, our results indicate that the presence of chemisorbed hydrogen 

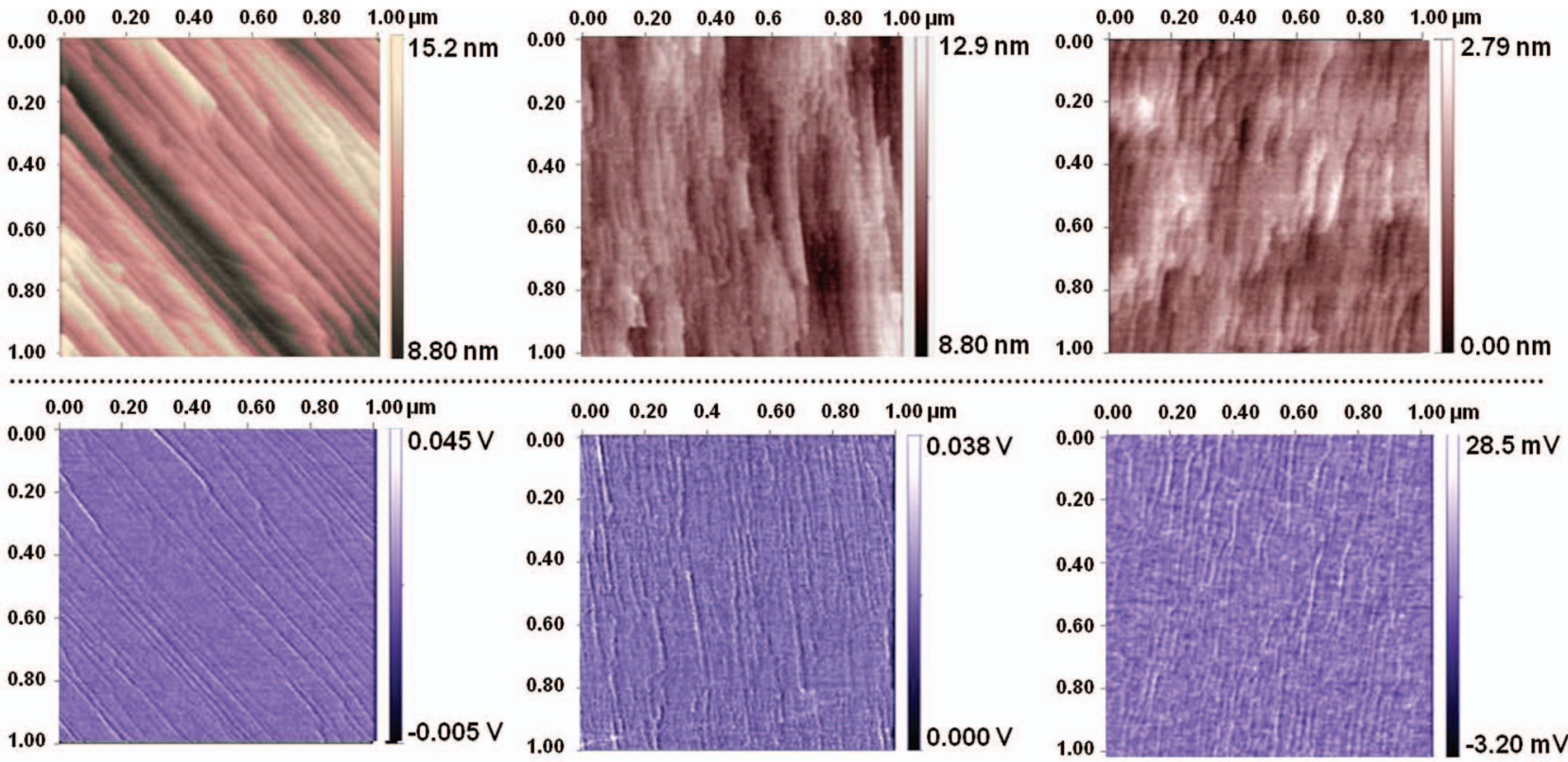

(a)

(b)

(c)

FIG. 1. Atomic force microscopy and friction force microscopy images of the GaAs (110) vicinal surface after deposition of $60 \mathrm{~nm}$ GaAs by (a) conventional MBE growth (sample I), (b) H-assisted oxide desorption followed by conventional MBE growth (sample II), and (c) H-assisted oxide desorption and growth (sample III). Image size: $1 \mu \mathrm{m} \times 1 \mu \mathrm{m}$.

lowers the surface friction and hence the surface energy with respect to the non-exposed GaAs (110) surface, an effect that is more significant after long $\mathrm{H}$ exposures, i.e., $\mathrm{H}$ coverage.

The observed variations in surface friction stem from the termination of surface atoms by different chemical species and the surface structural changes derived from it. The GaAs (110) surface, with equal numbers of $\mathrm{Ga}$ and As atoms, is the non-polar cleavage plane of zinc blende III-V semiconductors. It does not reconstruct, but exhibits a relaxation of the surface atomic positions, where the Ga atoms move inwards and the As atoms move outwards (Figure 2(a)), creating a characteristic $1 \times 1$ unit cell that is tilted by $25^{\circ}-27^{\circ}$ with respect to the ideal bulk surface (Figure 2(b)). ${ }^{18}$ The Ga and As surface atoms are both threefold coordinated; the $\mathrm{Ga}$ relaxes into an $\mathrm{sp}^{2}$ hybridization while the As acquires an $\mathrm{sp}^{3}$ hybridization and has a lone pair of electrons. The surface energy for the relaxed GaAs (110) has been calculated by several groups using either ab initio density-functional (50$\left.52 \mathrm{meV} / \AA^{2}\right)^{19,20}$ or tight-binding $\left(54 \mathrm{meV} / \AA^{2}\right)^{21}$ methods, in close agreement with the value extracted by Messmer $^{22}$ from fracture experiments, i.e., $54 \mathrm{meV} / \AA^{2}$. During the cooling process under the $\mathrm{As}_{4}$ beam that follows GaAs homoepitaxy by conventional MBE (sample I), As is adsorbed to a maximum coverage of one monolayer. Upon adsorption on the Ga surface sites the $\mathrm{Ga}$ atoms revert to the bulk $\mathrm{sp}^{3}$ configuration and move outwards, inducing a surface de-relaxation. By contrast, the As surface atoms do not relax significantly, staying practically in the same plane. ${ }^{23}$ The side view of the Asterminated GaAs (110) structure is depicted in Figure 2(c). This structure exists in thermodynamical equilibrium under As-stabilized conditions ${ }^{24}$ and has an associated surface energy of $45 \mathrm{meV} / \AA^{2}$, according to calculations by Moll and co-workers. ${ }^{19}$

During low temperature cleaning of sample II atomic $\mathrm{H}$ initially reacts with the GaAs surface native oxides, which are eliminated through the formation of volatile products. ${ }^{25}$ $\mathrm{H}$ chemisorbs non-preferentially on the Ga and As atoms of the clean surface, inducing coverage-dependent structural changes, which have been thoroughly studied using a variety of diffraction and spectroscopy techniques. ${ }^{26}$ It should be mentioned here that upon reaction the majority of hydrogen atoms are located above the substrate top layer and are

TABLE I. Raw and normalized value of the photodiode voltage obtained from FFM measurements on the as-grown GaAs (110) vicinal surface showing the effect of atomic hydrogen on the surface friction. The data presented for each sample are the average value obtained from six different scans. Also included are the estimated surface energy $(\sigma)$, the average terrace width $\left(\lambda_{0}\right)$, the Ga diffusion length $\left(\lambda_{G a}\right)$, the critical supersaturation ratio $\left(\alpha_{\text {crit }}\right)$, and the growth mode derived from Figure 3.

\begin{tabular}{lccccccc}
\hline \hline Sample & $\begin{array}{c}\Delta \mathrm{V}_{\mathrm{ph}} \\
(\mathrm{mV})\end{array}$ & $\begin{array}{c}\Delta \mathrm{V}_{\mathrm{ph}}(\mathrm{mV}) \\
(\text { normalized })\end{array}$ & $\begin{array}{c}\sigma \\
\left(\mathrm{meV} / \mathrm{A}^{2}\right)\end{array}$ & $\begin{array}{c}\lambda_{0} \\
(\mathrm{~nm})\end{array}$ & $\begin{array}{c}\lambda_{G a} \\
(\mathrm{~nm})\end{array}$ & $\begin{array}{c}\alpha_{\text {crit }} \\
\text { Growth mode }\end{array}$ \\
\hline I. Thermal oxide desorption and conventional MBE & $23 \pm 2$ & $1.0 \pm 0.1$ & 45 & $32.4 \pm 0.1$ & $\geq 16.20 \pm 0.05$ & $\ldots$ & $\begin{array}{c}\text { Step flow } \\
\text { II. H-assisted oxide desorption and conventional MBE }\end{array}$ \\
\hline III. H-assisted oxide desorption and H-MBE & $18.9 \pm 0.7$ & $0.82 \pm 0.03$ & $37 \pm 1$ & $31.6 \pm 0.1$ & $1.39 \pm 0.01$ & $2.40 \pm 0.01$ & Layer-by-layer \\
\hline \hline
\end{tabular}



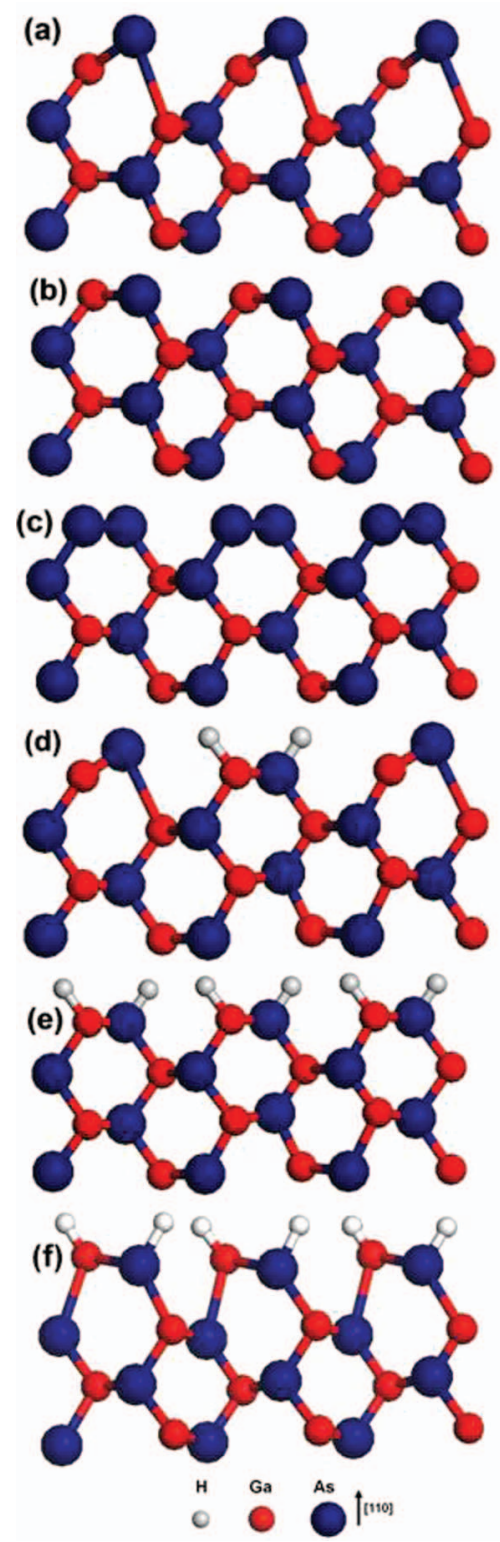

FIG. 2. Side views of the (a) relaxed, (b) ideal bulk, (c) As-terminated, (d) partially hydrogenated, (e) fully hydrogenated (de-relaxed), and (f) fully hydrogenated (counter-relaxed) GaAs (110) surface.

unable to diffuse into the subsurface layer, a fact verified by secondary ion mass spectrometry (SIMS) experiments performed on GaAs $(001)^{27}$ and $\operatorname{InP}(001)^{28}$ samples exposed to atomic deuterium and corroborated more recently by ion scattering spectroscopy time-of-flight (ISS-TOF) experiments on the hydrogenated (1 ML) GaAs(110) surface. ${ }^{26}$ With respect to the resulting structure, at low H coverages $(\leq 2000 \mathrm{~L})$ the non-covered fraction of the surface remains relaxed, but the surface $\mathrm{Ga}$ and As atoms bonded to $\mathrm{H}$ atoms recover their positions in the ideal structure, leading to a local de-relaxation of the surface, as depicted in Figure 2(d). Above the monolayer coverage the relaxation of the surface is removed ${ }^{29}$ and, under certain experimental conditions, the Ga-As chains in the first substrate layer rotate $-5^{\circ}$ leading to a slight counterrelaxation of the surface..$^{30}$ The existence of both structures has been predicted by theoretical calculations and confirmed experimentally, with the latter structure being found in gen- eral for very high $\mathrm{H}$ doses $(>5000 \mathrm{~L})$. A detailed description of these investigations can be found in Ref. 26. We have estimated that during the cleaning step the vicinal surface is exposed approximately to a $\mathrm{H}$ dose of $1200 \mathrm{~L}$. This means that the surface of the GaAs sample grown on the H-cleaned surface (sample II) is only partially relaxed, since $\mathrm{H}$ remains chemisorbed after the interruption of the $\mathrm{H}$ supply. Taking as a reference the theoretical energy value of the As-terminated GaAs (110) surface structure, i.e., $45 \mathrm{meV} / \AA^{2}$, which corresponds to sample I, we have calculated on the basis of the $18 \%$ reduction in $\Delta V_{p h}$ extracted from our FFM measurements that the partially $\mathrm{H}$-covered surface, present in sample II, has an energy value of $37 \mathrm{meV} / \AA^{2}$. In comparison, sample III is exposed to a higher dose of atomic $\mathrm{H}$ during its preparation, i.e., $3600 \mathrm{~L}$, as it is supplied to the surface continuously during surface cleaning and epitaxy. This dose is sufficient to reach the $\mathrm{H}$ monolayer coverage. By extrapolation of the FFM data extracted from sample III, we estimate that the surface energy of the fully hydrogenated GaAs (110) is $31 \mathrm{meV} / \AA^{2}$. The $\mathrm{H}$ doses used in this work thus result in H:GaAs (110) epilayers that are more stable than the relaxed clean surface (or the As-terminated GaAs(110)). In the absence of absolute values of the H:GaAs(110) surface energy in the literature, our data for the fully hydrogenated surface appear to be more consistent with a de-relaxation process in which the surface $\mathrm{Ga}$ and As bonded to $\mathrm{H}$ recover their position in the ideal bulk terminated surface, ${ }^{29}$ as depicted in Figure 2(e), rather than with a surface counter-relaxation ${ }^{30}$ like that shown in Figure 2(f), since stronger bonds are formed with hydrogen if the surface unrelaxes. In fact, the calculated energy gain in going from the relaxed surface structure to the counter-relaxed H:GaAs(110) structure reported in Ref. 30 is in clear contradiction with the stabilization observed for sample III.

The variations in the GaAs growth mode induced by the presence of atomic $\mathrm{H}$ were investigated at $620^{\circ} \mathrm{C}$ using the RHEED oscillation technique. Adatom migration and incorporation to step edges is favoured by the low growth rate and high temperature used in these experiments, which lead to step propagation during conventional MBE growth on the vicinal surface, in good agreement with the absence of oscillatory behaviour of the specular RHEED beam intensity shown in Figure 3(a). Accordingly, the AFM scan profiles corresponding to sample I did not evidence the presence of twodimensional islands on the terraces. The value of the Ga diffusion length can be estimated to be greater than half the terrace width $\left(\lambda_{\mathrm{Ga}}=\lambda_{0} / 2\right)$ measured for this sample, i.e., $\geq 16 \mathrm{~nm}$. By contrast, recording of RHEED oscillations at the onset of $\mathrm{GaAs}$ growth on the $\mathrm{H}$-cleaned surface and during $\mathrm{H}$-assisted growth (samples II and III, respectively) pointed to a layerby-layer mode, as depicted in Figure 3(b). From the delay to the first oscillation maximum ${ }^{31}$ on the vicinal $\mathrm{GaAs}(110)$ plane measured with respect to the singular surface we have calculated that almost $100 \%$ of the incoming Ga atoms are incorporated to the surface by 2D-nucleation on the terraces. This growth mode was corroborated by the observation of monolayer-height islands $\left(h_{(110)}=2 \AA\right)$ on the terraces of samples II and III by AFM. It was also found that the arsenic incorporation coefficient, $\mathrm{S}_{\mathrm{As} 4}$, determined for both samples II and III from the data in Figure 3 is 0.3 , which is approximately 

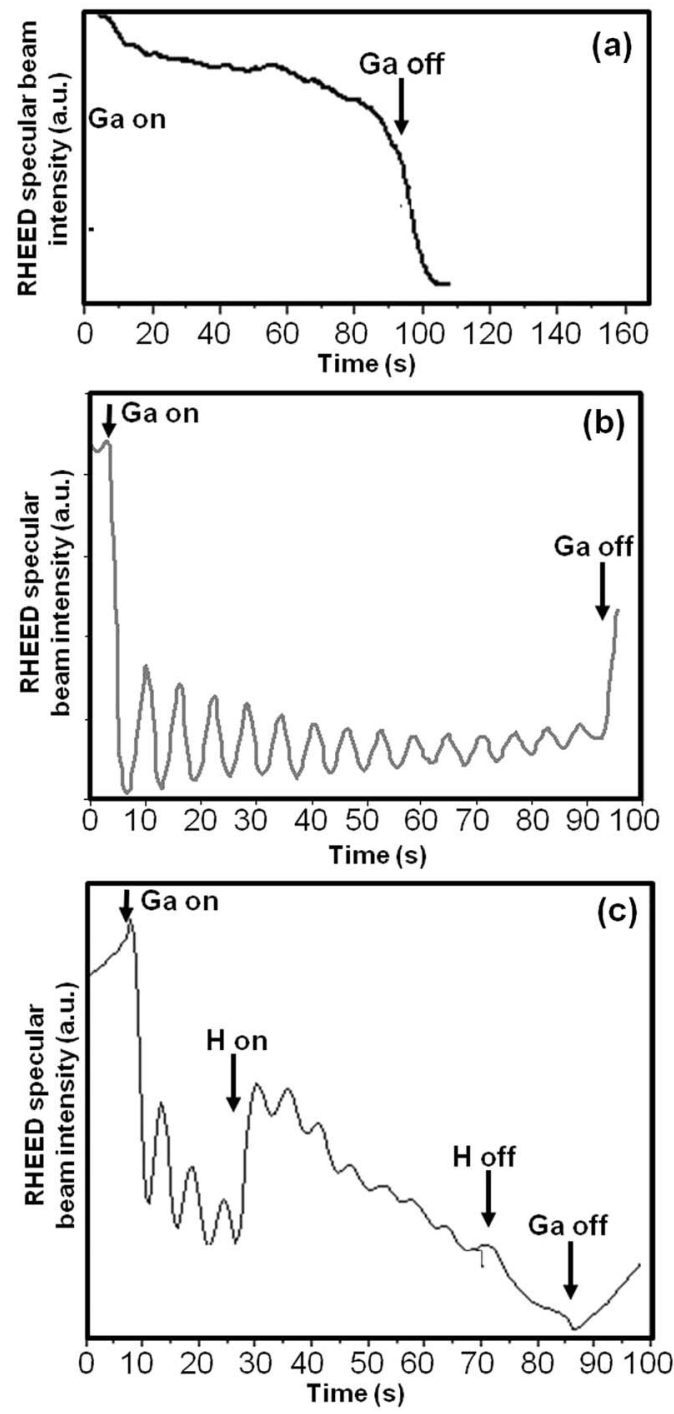

FIG. 3. RHEED specular beam intensity variation along the [110] azimuth of the vicinal GaAs (110) surface during the initial growth stages of (a) sample I, (b) sample II, and (c) sample III.

twice as high as that of the sample unexposed to atomic $\mathrm{H}^{32}$ as previously observed for low temperature GaAs growth. ${ }^{16}$ This result suggests that the surface lifetimes of the As species is longer on the H-terminated GaAs(110) surface, which helps to maintain the surface stoichiometry during growth. Moreover, it implies that the population of adsorbed As species has increased in the presence of atomic $\mathrm{H}$ and the more frequent adatom collisions result in an enhanced nucleation of $2 \mathrm{D}$ islands on the terraces. That is, the GaAs growth rate is artificially increased by the presence of the surfactant.

According to the classical theory of MBE growth of GaAs developed by Nishinaga, ${ }^{33}$ an increase in the growth rate can lead to a transition from step flow to $2 \mathrm{D}$ nucleation on the terraces of a vicinal surface when the adatom supersaturation ratio exceeds a critical value. If the desorption rate of $\mathrm{Ga}$ is negligible, which is the case at the typical temperatures used in GaAs MBE growth, the adatom concentration profile across a terrace can be approximated to a hyperbolic cosine function when growth proceeds by step propagation and chemical equilibrium at the steps is assumed. In this case the maximum and minimum values of the adatom concentration lie at the centre of the terrace and at the step edges, respectively. The maximum value of the supersaturation ratio on the terrace, $\alpha_{\max }$, is given by the product of the supersaturation ratios of $\mathrm{Ga}$ and $\mathrm{As}_{4}$, i.e., $\alpha_{\max }=\alpha_{\max }^{G a} \alpha_{\max }^{A s 4}$. But since the flux of $\mathrm{As}_{4}$ in our experiments is much higher than that of $\mathrm{Ga}$, we can assume that $\alpha_{\max }^{A s 4} \approx 1$ and, consequently, $\alpha_{\max }=\alpha_{\max }^{G a}$. Therefore, the maximum supersaturation ratio, found in the centre of the terrace $\left(\lambda_{0} / 2\right)$, can be expressed as

$$
\alpha_{\max }^{G a}=1+\frac{n_{0}}{2 \tau} \times\left(2 \pi m_{G a} k T\right)^{1 / 2} \frac{1}{P_{e}^{G a}} \times \frac{\lambda_{0}}{\lambda_{G a}} \tanh \frac{\lambda_{0}}{4 \cdot \lambda_{G a}} .
$$

In this equation $\lambda_{0}$ is the average terrace width, $\tau$ is the monolayer deposition time, $n_{0}$ is the density of lattice sites, $P_{e}^{G a}$ is the equilibrium vapour pressure of Ga under the specified growth conditions, $m_{G a}$ is the Ga atomic mass, $k$ is the Boltzman constant, and $T$ is the substrate temperature.

When $\alpha_{\max }$ exceeds a critical supersaturation ratio, $\alpha_{\text {crit }}$, 2D island nucleation on the terraces occurs. Hence, under the critical condition $\alpha_{\max }=\alpha_{\text {crit }}$ and Eq. (2) takes the form:

$$
\frac{\lambda_{0}}{4 \lambda_{G a}} \tanh \frac{\lambda_{0}}{4 \lambda_{G a}}=\frac{\tau}{2 n_{0}}\left(\alpha_{c r i t}-1\right) \times P_{e}^{G a} \times \frac{1}{\left(2 \pi m_{G a} k T\right)^{1 / 2}} .
$$

The expression of the critical supersaturation ratio can be derived from the classical nucleation theory through the calculation of the free energy required to form a critical disc-shape monolayer nucleus on the surface, ${ }^{34} W^{*}$ :

$$
W^{*}=-\frac{\pi \varepsilon^{2} \Omega}{h k T W_{V}},
$$

where $h, \varepsilon, \Omega$, and $W_{V}$ are the height of the nucleus, the nucleus edge energy per unit length, the atomic volume $(\Omega$ $=\mathrm{a}_{0}{ }^{3} / 8$ ), and the free energy required to form a cluster, respectively.

The critical supersaturation ratio is defined as $\alpha_{\text {crit }}$ $=\exp \left[-\frac{W_{V} \Omega}{k T}\right]$. Introducing the free energy for nuclei formation (Eq. (4)), $\alpha_{c r i t}$ can be expressed as

$$
\alpha_{c r i t}=\exp \left[\frac{\pi \varepsilon^{2} \Omega}{h k T W^{*}}\right] .
$$

The 2D nucleation rate $N$ is given by

$$
N=Z \omega^{*} n_{o} \exp \left(-W^{*} / k T\right) .
$$

Here $Z$ is the Zeldovich non-equilibrium factor to account for depletion of the population of critical nuclei due to their growth or decomposition and $\omega^{*}$ is the frequency with which the nuclei become supercritical.

Substituting $W^{*}$ from Eq. (6) into (5) and taking into account that $\varepsilon=h \sigma$, where $\sigma$ is the surface free energy, we find that

$$
\alpha_{c r i t}=\exp \left(\frac{\Omega \pi h \sigma^{2}}{\left(\ln \left(Z \omega^{*} n_{0}-\ln (N)\right) k^{2} T^{2}\right.}\right) .
$$

Hirth ${ }^{35}$ has shown that $Z \omega^{*} n_{o} \sim e^{65}$. The critical nucleation rate is generally assumed to be $10^{12} \mathrm{~cm}^{-2} \mathrm{~s}^{-1}$, which corresponds to one nucleation per unit time on a $10 \mathrm{~nm} \times 10 \mathrm{~nm}$ area (the average terrace widths in our experiments are $\approx 30 \mathrm{~nm}$ ). Accordingly, the critical supersaturation ratio can 
be expressed as

$$
\alpha_{c r i t}=\exp \left(\frac{\Omega \pi h \sigma^{2}}{\left(65-\ln 10^{12}\right) k^{2} T^{2}}\right) .
$$

Equations (3) and (8) can be applied to gain a better understanding of the change in adatom supersaturation conditions and $\mathrm{Ga}$ diffusion length associated with the transition to a dominant 2D layer-by-layer growth mode in the presence of atomic $\mathrm{H}$ observed for samples II and III once the surface free energy $(\sigma)$ has been determined from FFM measurements, while the height of the 2D islands $(h)$ and the average terrace width $\left(\lambda_{0}\right)$ have been extracted from AFM scan profiles. The average terrace width $\left(\lambda_{0}\right)$, in particular, is calculated from the terrace width distribution that results from 200 AFM scan profiles taken along the [001] direction for each of the topography images shown in Figure 1. The other parameters in Eqs. (3) and (8) are related to intrinsic properties of the GaAs material or to the specific growth conditions used. The calculated values of the critical supersaturation ratio, $\alpha_{\text {crit }}$, and the Ga diffusion length, $\lambda_{G a}$, for samples II and III are gathered in Table I. The data clearly indicate that the exposure to atomic hydrogen prior or during the homoepitaxy reduces significantly the Ga diffusion length on the GaAs(110) terraces, in accordance with the growth mode observed on the $\mathrm{H}$ terminated surface. Moreover, the $\lambda_{\mathrm{Ga}}$ value does not vary appreciably with the exposure time to the $\mathrm{H}$ beam, i.e., $\approx 1.4 \mathrm{~nm}$. The incoming $\mathrm{Ga}$ adatoms thus need to migrate a shorter length on the H-terminated terraces before they encounter a suitable reactive $\mathrm{AsH}_{\mathrm{x}}$ species ${ }^{6}$ to form GaAs. This higher encounter probability between $\mathrm{Ga}$ and As species must be the consequence of an enhanced $\mathrm{H}$-mediated dissociative chemisorption of the $\mathrm{As}_{4}$ molecules taking place prior to GaAs growth. Doubling of the arsenic incorporation coefficient on the H-terminated surface suggests that the As 4 dissociative chemisorption, which occurs via a pairwise interaction of $\mathrm{As}_{4}$ molecules adsorbed on adjacent Ga sites of the GaAs (110) surface, ${ }^{36}$ would likely involve a single $\mathrm{As}_{4}$ molecule that would react directly with the surface $\mathrm{H}$ atoms to form chemisorbed $\mathrm{AsH}_{\mathrm{x}}$ intermediate species. While this simplified reaction pathway needs to be confirmed by molecular beam mass spectroscopy studies, it appears as the most plausible explanation for the enhanced arsenic incorporation kinetics, leading to the shortened Ga adatom diffusion length observed.

\section{CONCLUSIONS}

We have investigated the modification of the surface energy and Ga diffusion length during homoepitaxial growth on vicinal GaAs (110) at $620^{\circ} \mathrm{C}$ in the presence of atomic $\mathrm{H}$ by FFM. It has been demonstrated that, despite the low sticking coefficient of $\mathrm{H}$ on the GaAs (110) surface at high temperatures, chemisorbed $\mathrm{H}$ used to remove the surface native oxides decreases the friction coefficient and hence the surface energy as the exposure to the $\mathrm{H}$ beam is prolonged over the epitaxy step, leading to more stable layers than those grown under Asstabilized conditions. The observed change in surface energy has been attributed to the progressive de-relaxation undergone by the surface with increasing $\mathrm{H}$ coverage, in comparison with the ideal bulk structure of the As-covered GaAs (110) surface grown by conventional MBE growth. In addition, the FFM data have shown a reduction of the Ga diffusion length, consistent with the change to a layer-by-layer growth mode observed by RHEED on the H-terminated GaAs (110) surface. The analysis of the RHEED intensity oscillations has allowed us to determine an arsenic incorporation coefficient during growth on the $\mathrm{H}$-covered surface that doubles its value on the non-exposed surface, which supports the hypothesis of a $\mathrm{H}$ mediated dissociative chemisorption of single $\mathrm{As}_{4}$ molecules with the formation of reactive $\mathrm{AsH}_{\mathrm{x}}$ intermediates.

\section{ACKNOWLEDGMENTS}

This work was supported by the Spanish MINECO under Grant No. MAT2011-22536.

${ }^{1}$ D. Kandel and E. Kaxiras, Solid State Phys. 54, 219 (1999).

${ }^{2}$ T. F. Kuech, AIP Conf. Proc. 916, 288 (2007).

${ }^{3}$ T. F. Wietler, E. Bugiel, and K. R. Hofmann, Appl. Phys. Lett. 87, 182102 (2005).

${ }^{4}$ J. Wollschläger, C. Deiter, C. R. Wang, B. H. Müller, and K. R. Hofmann, J. Appl. Phys. 110, 102205 (2011).

${ }^{5}$ V. Sih, R. C. Myers, Y. K. Kato, W. H. Lau, A. C. Gossard, and D. D. Awschalom, Nat. Phys. 1, 31 (2005).

${ }^{6}$ P. Tejedor, M. L. Crespillo, and B. A. Joyce, Appl. Phys. Lett. 88, 063101 (2006).

${ }^{7}$ P. Tejedor, L. Díez-Merino, I. Beinik, and C. Teichert, Appl. Phys. Lett. 95, 123103 (2009).

${ }^{8}$ L. Díez-Merino and P. Tejedor, J. Appl. Phys. 110, 013106 (2011).

${ }^{9}$ B. A. Joyce and T. B. Joyce, J. Cryst. Growth 264, 605 (2004) and references therein.

${ }^{10}$ E. S. Tok, J. H. Neave, J. Zhang, B. A. Joyce, and T. S. Jones, Surf. Sci. 374, 397 (1997).

${ }^{11}$ G. Hlawacek, Q. Shen, C. Teichert, A. Lex, G. Trimmel, and W. Kern, J. Chem. Phys. 130, 044703 (2009).

${ }^{12}$ H. Skulason and C. D. Frisbie, J. Am. Chem. Soc. 124, 15125 (2002).

${ }^{13}$ A. Noy, D. V. Venenov, and C. M. Lieber, "Chemical force microscopy," Annu. Rev. Mater. Sci. 27, 381 (1997).

${ }^{14}$ S. C. Clear and P. Nealey, Langmuir 17, 720 (2001).

${ }^{15}$ R. J. A. van den Oetelaar and C. F. J. Flipse, Surf. Sci. 384, L828-L835 (1997).

${ }^{16}$ P. Tejedor, M. L. Crespillo, and B. A. Joyce, Mater. Sci. Eng., C 26, 852 (2006).

${ }^{17}$ B. Galiana, M. Benedicto, and P. Tejedor, J. Appl. Phys. 113, 024309 (2013).

${ }^{18}$ A. Kahn, Surf. Sci. Rep. 3, 193 (1983).

${ }^{19}$ N. Moll, A. Kley, E. Pehlke, and M. Scheffler, Phys. Rev. B 54, 8844 (1996).

${ }^{20}$ R. Choudhury, D. R. Bowler, and M. J. Gillan, J. Phys.: Condens. Matter 20, 235227 (2008).

${ }^{21}$ M. Haugk, J. Elsner, and Th. Frauenheim, J. Phys.: Condens. Matter 9, 7305 (1997).

${ }^{22}$ C. Messmer and J. Bilello, J. Appl. Phys. 52, 4623 (1981).

${ }^{23}$ B. Kübler, W. Ranke, and K. Jacobi, Surf. Sci. 92, 519 (1980).

${ }^{24}$ J. E. Northrup, Phys. Rev. B 44, 1349 (1991).

${ }^{25}$ A. Muñoz-Yagüe, J. Piqueras, and N. Fabre, J. Electrochem. Soc. 128, 149 (1981).

${ }^{26}$ S. Nannarone and M. Pedio, Surf. Sci. Rep. 51, 1 (2003) and references within.

${ }^{27}$ D. E. Bliss, Ph.D. thesis (University of California, Berkeley, 1992), p. 61.

${ }^{28}$ R. R. LaPierre, B. J. Robinson, and D. A. Thompson, J. Cryst. Growth 191, 319 (1998).

${ }^{29}$ F. Manghi, C. M. Bertoni, C. Calandra, and E. Molinari, J. Vac. Sci. Technol. 21, 371 (1982).

${ }^{30}$ C. M. Bertoni, F. Finocchi, F. Bernardini, and M. Buongiorno Nardelli, Physica B 170, 429 (1991).

${ }^{31}$ T. Shitara, J. Zhang, J. H. Neave, and B. A. Joyce, J. Appl. Phys. 71, 4299 (1992). 
${ }^{32}$ D. M. Holmes, J. G. Belk, J. L. Sudijono, J. H. Neave, T. S. Jones, and B. A. Joyce, Appl. Phys. Lett. 67, 2848 (1995).

${ }^{33}$ T. Nishinaga and S. Naritsuka, Crystal Growth Technology (Springer-Verlag, Berlin, 2003), p. 55 and references therein; T. Nishinaga, Advances in Crystal Growth (Elsevier B.V., 2001), p. 110.
${ }^{34}$ D. Kashchiev and G. M. Rosmale, Cryst. Res. Technol. 38, 555 (2003).

${ }^{35}$ J. P. Hirth and G. M. Pound, Condensation and Evaporation, Progress in Material Science (The MacMillian Co., New York, 1963), p. 87.

${ }^{36}$ E. S. Tok, J. H. Neave, F. E. Allegretti, J. Zhang, T. S. Jones, and B. A. Joyce, Surf. Sci. 371, 277-288 (1997). 